TITLE:

\title{
In vivo structural analysis of articular cartilage using diffusion tensor magnetic resonance imaging
}

\section{$\operatorname{AUTHOR}(\mathrm{S})$ :}

Azuma, Takashi; Nakai, Ryusuke; Takizawa, Osamu; Tsutsumi, Sadami

\section{CITATION:}

Azuma, Takashi ... [et al]. In vivo structural analysis of articular cartilage using diffusion tensor magnetic resonance imaging. Magnetic Resonance Imaging 2009, 27(9): 1242-1248

\section{ISSUE DATE:}

2009-11

URL:

http://hdl.handle.net/2433/87131

\section{RIGHT:}

c 2009 Elsevier Inc. All rights reserved.; この論文は出版社版でありませ ん。引用の際には出版社版をご確認ご利用ください。; This is not the published version. Please cite only the published version. 
In vivo structural analysis of articular cartilage using diffusion tensor MRI

Takashi Azuma $^{1}$, Ryusuke Nakai ${ }^{1}$, Osamu Takizawa ${ }^{2}$ and Sadami Tsutsumi ${ }^{3}$

${ }^{1}$ Department of Medical Simulation Engineering,Research Center for Nano Medical Engineering, Institute for Frontier Medical Sciences, Kyoto University

${ }^{2}$ Siemens Asahi Medical Technologies Ltd, Medical Solutions Marketing Division,

${ }^{3}$ Nihon University School of Dentistry,

Contact:azuma@frontier.kyoto-u.ac.jp 


\section{Summary}

In vitro structure analysis of articular cartilage has recently been performed using diffusion tensor imaging (DTI) based on NMR imaging. We performed intravital imaging of human articular cartilage in vivo. Ultra-high-speed echo planar imaging (EPI) was optimized to provide images both in vivo and in vitro. While these images were obtained rapidly, and the resolution was lower than NMR, DTI of the structure of the articular cartilage was achieved. The structure was found to be different between images obtained in vivo vs. those obtained in vitro. The in vivo structural condition of the articular cartilage was elucidated. 


\section{Abstract}

Purpose: The articular cartilage is a small tissue with matrix structure of three layers between which the orientation of collagen fiber differs. A diffusion weighted twice-refocused spin echo EPI sequence was optimized for the articular cartilage and the structure of the three layers of human articular cartilage was imaged in vivo from diffusion tensor images.

Materials and Methods: The subjects imaged were 5 specimens of swine femur head after removal of the flesh around the knee joint, 5 specimens of swine articular cartilage with flesh present and the knee cartilage of five adult male volunteers. Based on diffusion-weighted images in 6 directions, the mean diffusivity (MD) and the fractional anisotropy (FA) values were calculated.

Results: Diffusion tensor images of the articular cartilage were obtained by sequence optimization. The MD and FA value of the specimens (each of five examples) under different conditions were estimated. Although the articular cartilage is a small tissue, the matrix structure of each layer in the articular cartilage was obtained by SE-EPI sequence with GRAPPA. Swine articular cartilage samples exhibited different MD and FA values of the articular cartilage in the synovial fluid and saline. In human articular cartilage the load of the body weight on the knee had an effect on the FA value of the surface layer of the articular cartilage. 
Conclusion: This method can be used to create images of the articular cartilage structure, not only in vitro but also in vivo. Therefore, it is suggested that this method supports the elucidation of the in vivo structure and function of the knee joint and might be applied to clinical practice. 


\section{Introduction}

Diffusion-weighted imaging (DWI) allows for noninvasive measurement of Brownian motion of water molecules in vivo, and is different from magnetic resonance imaging (MRI) (T1- and T2-weighted images). Using information of Brownian motion of water molecules, microscopic cellular structure can be imaged[1,2]. Water molecules in vivo are prevented from free diffusion by the cellular membrane and fibers, i.e., the directions that are easy and difficult to diffuse are found and diffusion anisotropy has been studied in vivo [3-8]. Diffusion tensor imaging (DTI) is a method of examining and expressing diffusion anisotropy [9-11]. Many researchers have studied DTI in imaging of the central nervous system [12-15].

In this study, the structure of the fibrous structure of the articular cartilage was analyzed using DTI. The articular cartilage contains no blood or lymph vessels or nerves and comprises rough chondrocytes and a rich cartilage matrix. To be specific, it is composed of $60-80 \%$ water and $20-40 \%$ matrix with a small volume of chondrocytes. Regarding the biological structure, the articular cartilage is a hyaline cartilage composed of three layers, the surface, middle and deep parts, and maintains lubrication by water contained in the matrix and by the different degrees and directions of collagen fiber orientation of each layer. The matrix is composed of collagen and proteoglycan, which is a high-molecular-weight polymer of proteoglycan and amino sugar connected with hyaluronic acid. Proteoglycan retains a large volume of water, consequently [16]. In contrast, collagen is a structural protein that plays a role in maintaining and building up the form of cartilage, functioning as the matrix structure of the articular cartilage. The structure of cartilage 
is comprised of three layers, the surface, middle and deep regions [16-20]. The orientation and density of collagen differ between layers. The synovial fluid containing the matrix structure, which is assembled from collagens with different orientation and density, and proteoglycan gel (polymer), which retains a large volume of water, thick hyaluronan and chondroitin sulfate, functions as a shock absorber and lubricant, which are characteristics of the articular cartilage.

The synovial fluid containing the matrix structure that is assembled from collagens with different orientation and density, and proteoglycan gel (polymer) that retains a large volume of water, functions as a shock absorber and lubricant, which are the main characteristics of the articular cartilage. Therefore, in imaging of the articular cartilage, it is important to image the condition of the matrix structure. This structural anisotropy of each layer is an important factor that defines the function of the cartilage. Approaches towards regenerative treatment of the articular cartilage have recently been made for patients with osteoarthritis of the knee[21]. As such, tissues without a cartilage layer structure may be treated, however, it is necessary to diagnose the morphology of the articular cartilage by imaging and assess the structure of each layer. Therefore, estimation of the structural anisotropy, which is deeply involved in the function of the cartilage, is essential for functional evaluation of regenerative treatment for defect(s) of the articular cartilage.

Recent studies have examined diffusion of the articular cartilage using MRI [22-25]. In addition, basic in vitro studies on DTI in articular cartilage by nuclear magnetic resonance (NMR) analysis have also been performed [26-29]. We analyzed the structure of the articular cartilage of swine knee joint in vitro and that of normal human articular cartilage of the knee joint using 
optimized SE-EPI diffusion sequence, ultrafast imaging $[6,30]$. 


\section{Materials and Methods}

MR imaging

In an in vitro study using the cartilage of an animal knee joint, which was provided from an abbatoir, a resected part of the swine knee joint from approximately $150 \mathrm{~mm}$ below to $150 \mathrm{~mm}$ above the knee was imaged up to 24 hours after resection. (a) The flesh around the knee joint was removed and five specimens of femur heads alone soaked in saline were imaged. (b) Five specimens of the knee joint surrounded by the intact flesh post-resection were imaged. In a study of the cartilage of human knee joints, (c) five healthy adult male volunteers laid on the MRI bed after standing for about ten minutes and the cartilage of the knee joint was imaged 3 minutes later. The cartilage of knee joint was imaged again after the subjects continued to lie in bed for 60 minutes.

MRI with a homemade four-channel flexarray receiving-coil was conducted using Sonata $1.5 \mathrm{~T}$

(Siemens, Erlangen, Germany). To avoid artifact due to movement, the DWI sequence was optimized using SE-EPI (spin echo EPI) ultrafast imaging sequence. To reduce distortion of the image due to eddy currents generated by a motion probing gradient (MPG) of the DWI, a diffusion weighted twice-refocused spin echo EPI sequence was used [31].

The width of the articular cartilage was approximately 3-5 $\mathrm{mm}$. Consequently, the acquisition matrix of the data in the direction encoding frequency and phase was increased to obtain a high-resolution image. However, an increase in the acquisition matrix prolongs the echo reading time and enhances image distortion. Therefore, using an integrated parallel imaging (iPAT) method 
the echo reading time was shortened and image distortion was reduced [32].

Images were taken using a DWI $b$ factor of $300-1000 \mathrm{sec} / \mathrm{mm}^{2}$. In the above range, a sufficient diffusion contrast was obtained in human articular cartilage at $600 \mathrm{sec} / \mathrm{mm}^{2}$, therefore, $600 \mathrm{sec} / \mathrm{mm}^{2}$ was used as the DWI $\mathrm{b}$ factor. The sagittal section was imaged under the following conditions: TR: 2200ms, TE: 73ms, Field of view (FOV): 192*192, Matrix: 192*19, Averages: 23, Scan time: 6min 48s, MPG: 6 axes, b factor: 600s/ $\mathrm{mm}^{2}$, Thickness: $5 \mathrm{~mm}$, iPAT: GeneRalized Autocalibrating Partially Parallel Acquisitions (GRAPPA) Acceleration Factor PE (phase encoding) 2 and Reference Lines PE (phase encoding) 25. Herein, the six directions of MPG are $(\mathrm{x}, \mathrm{y}, \mathrm{z})=(1,0,1)$, $(-1,0,1),(0,1,1),(0,-1,1),(1,1,0)$ and $(-1,1,0)$.

Analysis of diffusion tensor imaging (DTI)

Using DW images (b0 image) at $\mathrm{b}$ factor of $0 \mathrm{sec} / \mathrm{mm}^{2}$ and those of six directions in MPG, diffusivity at six directions (apparent diffusion constant: ADC) was estimated. DTI (1) was estimated with the above diffusivity [15].

$$
\mathbf{D}=\left(\begin{array}{lll}
D_{x x} & D_{x y} & D_{x z} \\
D_{y x} & D_{y y} & D_{y z} \\
D_{z x} & D_{z y} & D_{z z}
\end{array}\right)
$$

Next, DTI (1) was triply diagonalized using Householder transformation. Furthermore, using 
the QL(Q: orthogonal Matrix, L : Lower Triangular Matrix) method, eigenvalues $(\lambda 1, \lambda 2, \lambda 3)(2)$ and eigenvectors $(e 1, e 2, e 3)(3)$ were calculated from the $\mathrm{Q}$ and $\mathrm{L}$ matrices. Eigenvalues $(\lambda 1, \lambda 2$, $\lambda 3$ ) correspond to the diffusion intensity.

$$
\mathbf{P D P}^{T}=\left(\begin{array}{ccc}
\lambda_{1} & 0 & 0 \\
0 & \lambda_{2} & 0 \\
0 & 0 & \lambda_{3}
\end{array}\right) \quad \lambda_{1}>\lambda_{2}>\lambda_{3} \quad(2) \quad \mathbf{P}=\left(\begin{array}{c}
e_{1}^{T} \\
e_{2}^{T} \\
e_{3}^{T}
\end{array}\right)
$$

The eigenvector $e 1$ corresponding to the eigenvector $\lambda 1$ represents a vector of the direction in which diffusion is maximum. To be specific, this $e 1$ vector indicates the direction most easily diffusible in the calculated pixels. Using these eigenvalues, the mean diffusivity (MD) (4) was calculated [15].

$$
M D=\bar{\lambda}=\frac{\lambda_{1}+\lambda_{2}+\lambda_{3}}{3}
$$

Using these eigenvalues, fractional anisotropy (FA), a value indicating the degree of water diffusion, was estimated. The equation to calculate the FA is shown below (5) [15].

$$
F A=\sqrt{\frac{3}{2}} \sqrt{\frac{\left(\lambda_{1}-\bar{\lambda}\right)^{2}+\left(\lambda_{2}-\bar{\lambda}\right)^{2}+\left(\lambda_{3}-\bar{\lambda}\right)^{2}}{\lambda_{1}^{2}+\lambda_{2}^{2}+\lambda_{3}^{2}}}
$$


Data analysis

The image of the MR data of two kinds $\{(\mathrm{a}) 、(\mathrm{~b})\}$ of swine articular cartilages and two kinds $\{(c-3) 、(c-60)\} \quad$ of human articular cartilage was analyzed. (a) 5 swine articular cartilages after removal of flesh, (b) 5 swine articular cartilage with flesh intact, (c) five adult male volunteers, i.e., (c-3) human articular cartilage after 3-minute bed rest, and (c-60) human articular cartilage after 60-minute bed rest. And based on the calculation of (1) - (5), MD and FA values were analyzed by a specific calculation program that was created. For each DWI image, the anterior sagittal section was measured and the MD and FA values between the surface of the articular cartilage and the subchondral bone interface were calculated. The measurement between the surface of the articular cartilage and the subchondral bone interface was conducted at 9 equidistant points and the MD and FA values were calculated using bilinear transformation of 4 vicinal pixels. The measurements were performed manually and the measurement points are shown in Figure.1. 


\section{Results}

Figure. 1 presents each of seven DWI images ( $b$ factor $=0$ and $b=600 \mathrm{~s} / \mathrm{mm}^{2}$ ) of (a), (b) and (c-3). Each typical MD map and FA maps were shown in Figure2. The mean MD and FA values of 5 samples at 9 points between the surface and deep layer of the articular cartilage of the front portion of the knee (Measurement point in Figure. 1) of (a), (b) and (c-3) and their standard deviations are shown in Figure 3 and Table 1 and Table 2, respectively. Similarly, the mean MD and FA values of (c-3) and (c-60) and their standard deviations of 5 samples are shown in Figure 4 and Table 1 and Table 2, respectively. In Figures 3 and 4, the vertical axes indicate MD=0.0 as brightness $=0$ and $\mathrm{MD}$ maximum as brightness $=255$, whereas, the horizontal axes indicate the depth from the surface in proportion to the cartilage surface of 0 and the subchondral bone of 100 . Similarly, the vertical axes indicate $\mathrm{FA}=0.0$ as brightness $=0$ and $\mathrm{FA}=1.0$ as brightness $=255$, while the horizontal axes indicate the depth from the surface in proportion to the cartilage surface of 0 and the subchondral bone of 100 . The data representing $10 \%$ of the articular cartilage surface are on the critical pixels and the partial volume effect can occur; therefore, examination within this region was excluded from this study.

In Figure 3 the absolute MD values at (a), (b) and (c-60) differed; however, graphs at these points showed a similar tendency. In the three samples, FA values from the deep to the middle layers were decreased; in contrast, the values were high at (b) and (c-60) from the middle to the surface layers. At the points of (a), (b) and (c-60) of the surface of the articular cartilage, MD values were slightly decreased and FA values were increased in saline, but not at the point of (a). The MD and FA values 
of the articular cartilage at the points of (a) and (b) were different between the synovial fluid and saline., In sample (a) of this study, which Q6 was studied using articular cartilage soaked in saline, which is similar to in vitro studies using spin echo [26-28], the results were similar to the results of a NMR study by Meder et al. [27]. The MD value was the highest at the surface of the articular cartilage and decreased gradually in the middle and deep layers. On the other hand, the FA value was the highest in the deep layer of the articular cartilage and decreased gradually in the middle layer and surface. Using DWI sequence ultrafast imaging by parallel imaging, the structure of articular cartilage could be imaged. Although the articular cartilage is a small tissue, it could be imaged by DWI sequence ultrafast imaging technique.

At the points of (c-3) and (c-60) of human articular cartilage, MD values at (c-60) of the surface were lower than those at $(\mathrm{c}-3)(\mathrm{p}<0.05)$ while FA values were virtually similar (Figure 3$)$. These results show that the load of the body weight on the knee had an effect on the MD value of the articular cartilage, i.e., the extent of diffusion.

In Figure 4 at points (c-3) and (c-60) of human articular cartilage, the MD values at (c-3) were similar to those at (c-60) while the FA values were almost similar in the deep layer but slightly higher in the middle and surface layers. A possible cause of the difference in the FA values between the images on the same section, 3 and 60 minutes after the volunteers laid on the bed from the standing position and their knees were fixed, is that the weighted cartilage of the knee joint was gradually released as time passed. 


\section{Discussion}

The articular cartilage is composed of hyaline cartilage and the width range is approximately 3-5

mm. T2 relaxation time ranged $20-60 \mathrm{~ms}[33,34]$. The articular cartilage is composed of $60-80 \%$ synovial fluid and $20-40 \%$ matrix with a small volume of chondrocytes. The synovial fluid consists of mainly thick hyaluronan and chondroitin sulfate.

The articular cartilage of sample (a) was placed in saline while that of sample (b) was placed in the synovial fluid. The reason the MD values at (a) were higher than those at (b) in Figure. 3 is likely because saline is more diffusible than the gelatinous synovial fluid. The FA values at (a) from the deep to the surface layers were monotonically decreased, whereas the FA values at (b) decreased from the deep to middle layers and conversely increased from the middle to surface layers. This result was also caused by differences in the status around the articular cartilage between the synovial fluid and saline. In the synovial fluid, the water component contained both the synovial fluid (gel) and proteoglycan in equilibrium within the articular cartilage, whereas, in saline, the water component flowed in the articular cartilage more than that in the synovial fluid. Consequently, the surface of the articular cartilage was swollen, resulting in decreased FA values. Based on the fact that the difference between MD values at (a) and (b) of the surface was larger than that of the deep region, it is likely that the surface of the articular cartilage was swollen.

Figure. 4 indicates that the weight loaded on the knees was released over time. The differences in FA values from the middle to surface layers depend on the structure of the articular cartilage. Specifically, collagen fibers from the middle to deep layers are oriented parallel to the weight 
direction and are virtually unaffected by the weight, on the other hand, in the middle layer, collagen fibers are oriented in an oblique direction to the weight direction and are slightly affected by the weight. Furthermore, in the surface layer, collagen fibers are oriented orthogonally to the weight direction, and are consequently, significantly affected by the weight. Therefore, the structure of collagen fibers in the middle and surface layers, which are easily affected by the weight, became more dense due to the weight, which led to the increased FA values.

Similar results were obtained from a study by de Visser et al., i.e., NMR imaging with weighted articular cartilage samples [29].

As shown in Figure 2, the difference between (a) and (b) is due to the difference between the synovial fluid and saline.

The synovial fluid and the general structure of the articular cartilage functions well to absorb shock. Therefore, further studies should be performed to elucidate the relationship between the structure of the articular cartilage and the synovial fluid.

In the synovial fluid, protons are captured by proteoglycan and motion is limited. On the other hand, in saline, proton motion is not suppressed and protons can easily infiltrate into collagen fibers in the articular cartilage. Consequently, in (a), the MD value was the highest at the surface of articular cartilage and decreased gradually in the middle and deep layers, and the FA value was the highest in the deep layer of articular cartilage and decreased gradually in the middle layer and surface. These results suggest that in absorbing shock, the surface of articular cartilage functions most effectively as a shock absorber because water influx and efflux is easiest at the surface, and 
thus, the density of the articular structure is easily altered.

In this study, the matrix structure image of each layer in the articular cartilage was obtained in vivo by ultrafast imaging using weighted twice-refocused spin echo EPI diffusion, and it was shown that the synovial fluid and the surface of the articular cartilage layer structure played an important role in the knee articulation, which required both motor function and shock-absorbing function. In human imaging, it is necessary to consider the effect of blood flow. The diffusion image exhibited the effects of blood vessel movement and the influence of synovial fluid, and they generated artifacts. At a b factor of approximately $500 \mathrm{sec} / \mathrm{mm}^{2}$ and less, clear diffusion images were difficult to obtain due to blood vessel movement and the effect of the synovial fluid. By contrast, at $\mathrm{a} b$ factor of approximately $900 \mathrm{sec} / \mathrm{mm}^{2}$ and more, the $\mathrm{S} / \mathrm{N}$ signal-to-noise ratio

in the image was not suitable. Consequently, the optimal $b$ factor ranged from 600-700 $\mathrm{sec} / \mathrm{mm}^{2}$. Further establishment of this method in future could be applied to the clinical diagnosis of knee osteoarthritis and cartilage regeneration therapy [35]. 


\section{References}

[1] Stejskal EO, Tanner JE. Spin Diffusion Measurements - Spin Echoes in Presence of a Time-Dependent Field Gradient. Journal of Chemical Physics 1965;42 (1):288-92.

[2] Le Bihan D, Breton E, Lallemand D, Grenier P, Cabanis E, Laval-Jeantet M. MR imaging of intravoxel incoherent motions: application to diffusion and perfusion in neurologic disorders. Radiology 1986;161 (2):401-7.

[3] Cleveland G, G., Chang D, C., Hazelwood C, F. Nuclear magnetic resonance measurements of skeletal muscle. Anisotropy of the diffusion coefficient of the intracellular water. Biophys J 1976; 16 (1043-1053)

[4] Moseley ME, Cohen Y, Kucharczyk J. Diffusion-weighted MR imaging of anisotropic water diffusion in cat central nervous system. Radiology 1990;176:439-46.

[5] Chenevelt T, L., Brunberg J, A., Pipe JG. Anisotropic diffusion within human white matter: demonstration with NMR techniques in vivo. Radiology 1990;177:401-5.

[6] Turner R, Le Bihan D, Maier J. Echo-planar imaging of intravoxel incoherent motions. Radiology 1990;177:407.

[7] Lythgoe M, F., Busza A, L., Calamante F. Effects of diffusion anisotropy on lesion delineation in a rat model of cerebral ischemia. Magn Reson Med 1997;38:662-8.

[8] Hoehn Berlage M, Eis M, Schmitz B. Regional and directional anisotropy of apparent diffusion coefficient in rat brain. NMR Biomed 1999;12:45-50.

[9] Douek P, Turner R, Pekar J, Patronas N, J., Le Bihan D. MR color mapping of myelin fiber orientation. J Comput Assist Tomogr 1991;15:923-9.

[10] Basser PJ, Mattiello J, LeBihan D. Estimation of the effective self-diffusion tensor from the NMR spin echo. J Magn Reson B 1994;103 (3):247-54.

[11] Basser PJ, Mattiello J, Le Bihan D. MR diffusion tensor spectroscopy and imaging. Biophys J 1994;66:259-67.

[12] Pierpaoli C, Jezzard P, Basser PJ, Barnett A, Di Chiro G. Diffusion tensor MR imaging of the human brain. Radiology 1996;201 (3):637-48.

[13] Basser PJ, Pierpaoli C. A simplified method to measure the diffusion tensor from seven MR images. Magn Reson Med 1998;39 (6):928-34.

[14] Jones DK, Horsfield MA, Simmons A. Optimal strategies for measuring diffusion in anisotropic systems by magnetic resonance imaging. Magn Reson Med 1999;42 (3):515-25.

[15] Le Bihan D, Mangin JF, Poupon C, Clark CA, Pappata S, Molko N, Chabriat H. Diffusion tensor imaging: concepts and applications. J Magn Reson Imaging 2001;13 (4):534-46.

[16] Buckwalter JA, Mankin HJ. Articular cartilage: tissue design and chondrocyte-matrix interactions. Instr Course Lect 1998;47:477-86.

[17] Clarke IC. Articular cartilage: a review and scanning electron microscope study. 1. The interterritorial fibrillar architecture. J Bone Joint Surg Br 1971;53 (4):732-50.

[18] Glaser C, Putz R. Functional anatomy of articular cartilage under compressive loading Quantitative aspects of global, local and zonal reactions of the collagenous network with 
respect to the surface integrity. Osteoarthritis Cartilage 2002;10 (2):83-99.

[19] Le Bihan D. Intravoxel incoherent motion imaging using steady-state free precession. Magn Reson Med 1988;7 (3):346-51.

[20] Nieminen MT, Rieppo J, Toyras J, Hakumaki JM, Silvennoinen J, Hyttinen MM, Helminen HJ, Jurvelin JS. T2 relaxation reveals spatial collagen architecture in articular cartilage: a comparative quantitative MRI and polarized light microscopic study. Magn Reson Med 2001;46 (3):487-93.

[21] Wakitani S, Goto T, Young RG, Mansour JM, Goldberg VM, Caplan AI. Repair of large full-thickness articular cartilage defects with allograft articular chondrocytes embedded in a collagen gel. Tissue Eng 1998;4 (4):429-44.

[22] Burstein D, Gray ML, Hartman AL, Gipe R, Foy BD. Diffusion of small solutes in cartilage as measured by nuclear magnetic resonance (NMR) spectroscopy and imaging. J Orthop Res 1993;11 (4):465-78.

[23] Xia Y, Farquhar T, Burton-Wurster N, Vernier-Singer M, Lust G, Jelinski LW. Self-diffusion monitors degraded cartilage. Arch Biochem Biophys 1995;323 (2):323-8.

[24] Knauss R, Schiller J, Fleischer G, Karger J, Arnold K. Self-diffusion of water in cartilage and cartilage components as studied by pulsed field gradient NMR. Magn Reson Med 1999;41 (2):285-92.

[25] Mlynarik V, Sulzbacher I, Bittsansky M, Fuiko R, Trattnig S. Investigation of apparent diffusion constant as an indicator of early degenerative disease in articular cartilage. J Magn Reson Imaging 2003;17 (4):440-4.

[26] Filidoro L, Dietrich O, Weber J, Rauch E, Oerther T, Wick M, Reiser MF, Glaser C. High-resolution diffusion tensor imaging of human patellar cartilage: feasibility and preliminary findings. Magn Reson Med 2005;53 (5):993-8.

[27] Meder R, de Visser SK, Bowden JC, Bostrom T, Pope JM. Diffusion tensor imaging of articular cartilage as a measure of tissue microstructure. Osteoarthritis Cartilage 2006;14 (9):875-81.

[28] Deng X, Farley M, Nieminen MT, Gray M, Burstein D. Diffusion tensor imaging of native and degenerated human articular cartilage. Magn Reson Imaging 2007;25 (2):168-71.

[29] de Visser SK, Crawford RW, Pope JM. Structural adaptations in compressed articular cartilage measured by diffusion tensor imaging. Osteoarthritis Cartilage 2007;

[30] Stehling MK, Turner R, Mansfield P. Echo-planar imaging: magnetic resonance imaging in a fraction of a second. Science 1991;254 (5028):43-50.

[31] Reese TG, Heid O, Weisskoff RM, Wedeen VJ. Reduction of eddy-current-induced distortion in diffusion MRI using a twice-refocused spin echo. Magn Reson Med 2003;49 (1):177-82.

[32] Griswold MA, Jakob PM, Heidemann RM, Nittka M, Jellus V, Wang J, Kiefer B, Haase A. Generalized autocalibrating partially parallel acquisitions (GRAPPA). Magn Reson Med 2002;47 (6):1202-10.

[33] Mlynarik V, Trattnig S, Huber M, Zembsch A, Imhof H. The role of relaxation times in 
monitoring proteoglycan depletion in articular cartilage. J Magn Reson Imaging 1999;10 (4):497-502.

[34] Mosher TJ, Dardzinski BJ, Smith MB. Human articular cartilage: influence of aging and early symptomatic degeneration on the spatial variation of T2--preliminary findings at $3 \mathrm{~T}$. Radiology 2000;214 (1):259-66.

[35] Buckwalter JA, Mankin HJ. Articular cartilage: degeneration and osteoarthritis, repair, regeneration, and transplantation. Instr Course Lect 1998;47:487-504. 


\section{Figure}

(a)
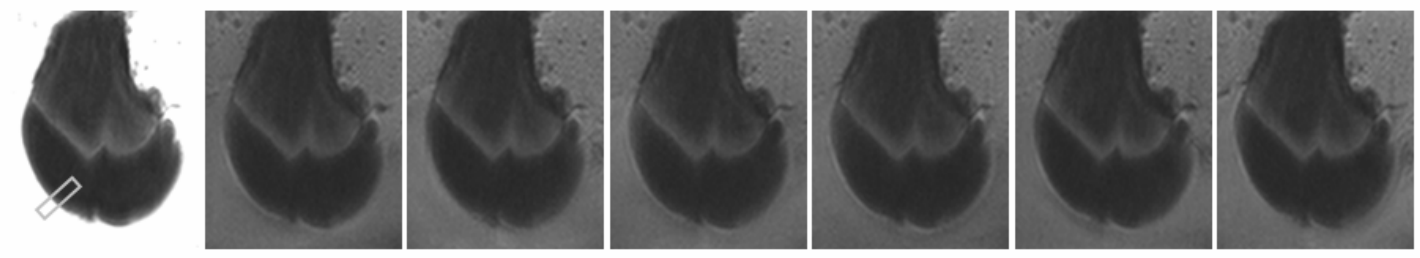

(b)
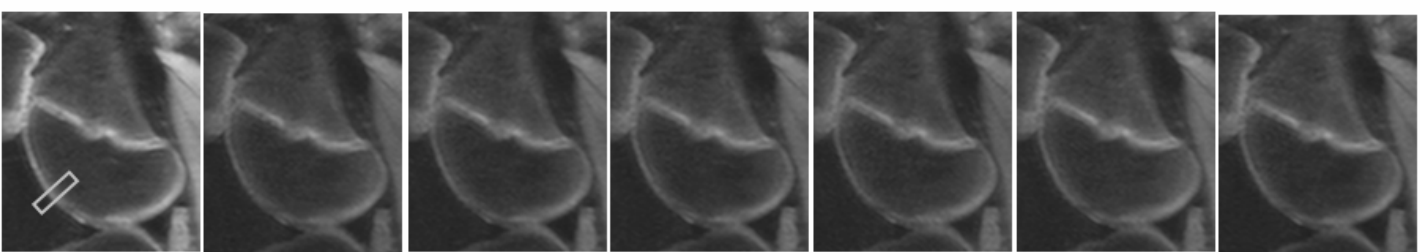

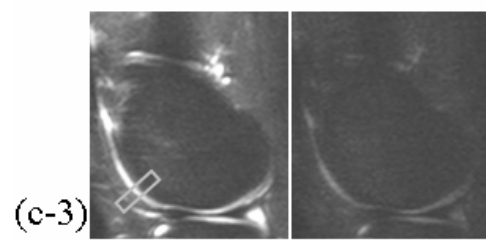

B

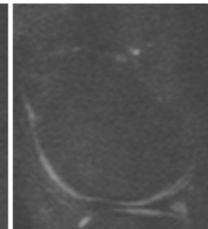

C

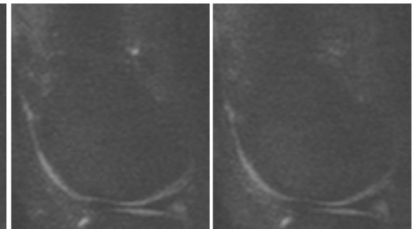

D

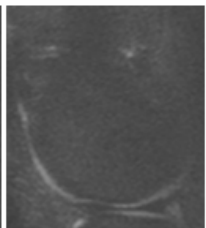

F

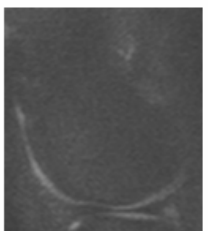

G

Figure.1.

Figure 1. Diffusion weighted images by optimized twice refocused spin echo EPI diffusion sequence: (a) swine articular cartilage after removal of the flesh, (b) swine articular cartilage with the flesh intact, and (c-3) human articular cartilage after 3-minute bed rest. Diffusion-weighted images using MPG at six directions at $b=0 \mathrm{~s} / \mathrm{mm}^{2}$ (A) and $b=600 \mathrm{~s} / \mathrm{mm}^{2}$ (B-G). Six directions of MPG are $(x, y, z)=B(1,0,1), C(-1,0,1), D(0,1,1), E(0,-1,1), F(1,1,0)$ and $G(-1,1,0)$. 
(a)
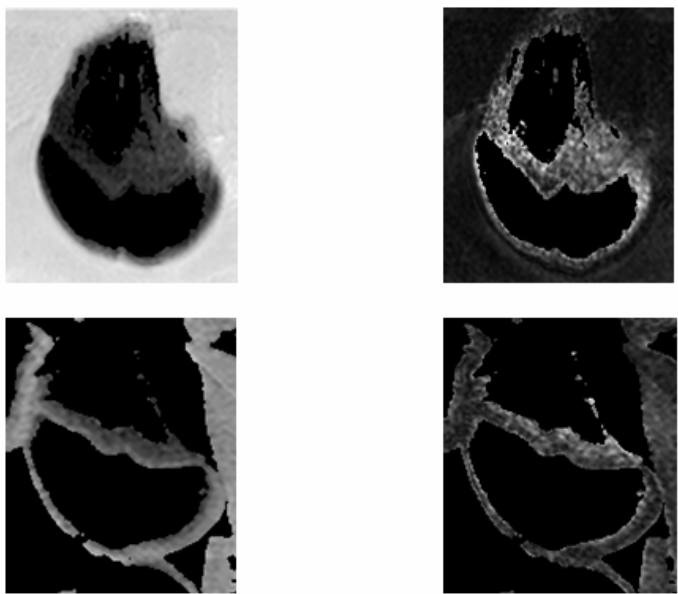

(b)
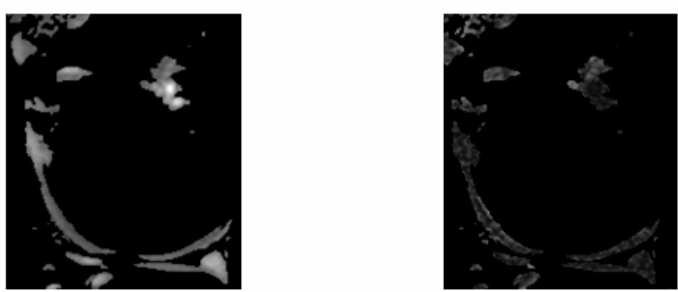

Mean diffusivity map Fractional anisotropy map

Figure.2.

Figure 2. Typical MD maps (a), (b) and (c-3) and FA maps are shown in Figure2.

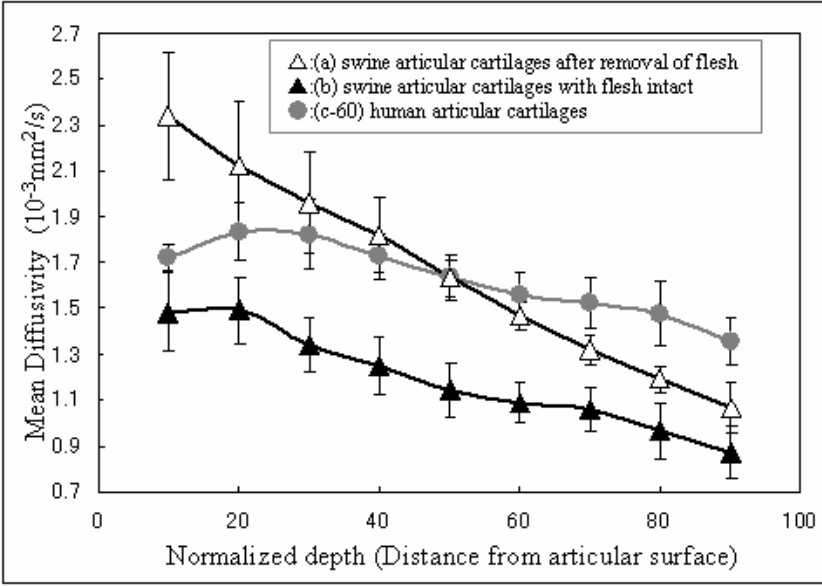

(A) Mean diffusivity

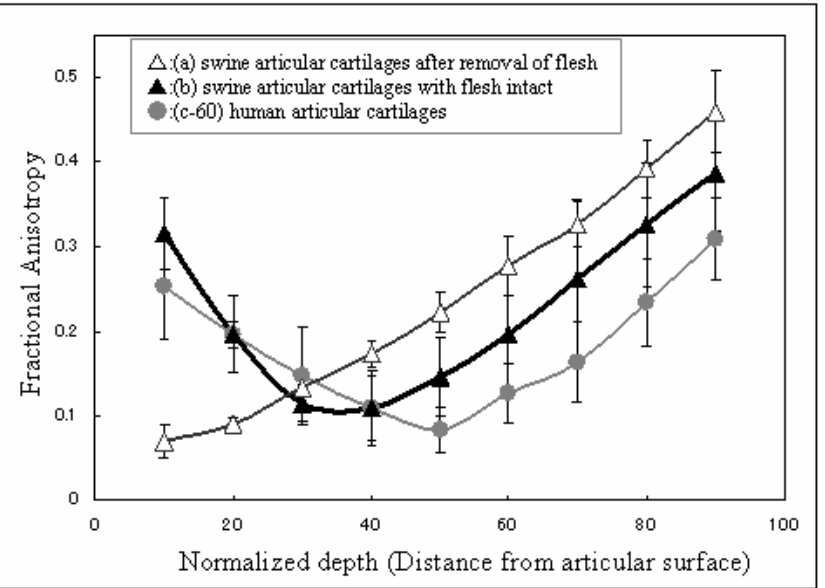

(B) Fractional anisotropy values

Figure.3.

Figure 3. (A) (c-60): Human articular cartilage after 60 minutes of bed rest. The mean MD values and their standard deviations in the region between the surface and deep layer of the articular cartilage of (a), (b) and (c-60) are shown. The vertical axis indicates $\mathrm{MD}=0.0$ as brightness $=0$ and MD maximum as brightness $=255$, on the other hand, the horizontal axis indicates the depth from the surface in proportion to the cartilage surface (0) and the subchondral bone interface (100). The 
absolute MD values at (a), (b) and (c-60) differed; however, graphs at these points showed a similar tendency.

(B) The mean FA values and their standard deviations in the region between the surface and deep layer of the articular cartilage of (a), (b) and (c-60) are shown. The vertical axis indicates FA=0.0 as brightness $=0$ and $\mathrm{FA}=1.0$ as brightness $=255$, on the other hand, the horizontal axis indicates the depth from the surface in proportion to the cartilage surface $(0)$ and the subchondral bone interface (100). The FA value of (a) was the highest in the deep layer of articular cartilage and decreased gradually in the middle layer and surface. Similar to Figure 3-(A), (b) and (c-60), in contrast to (a), the FA value was higher from the middle to the surface layers of articular cartilage.

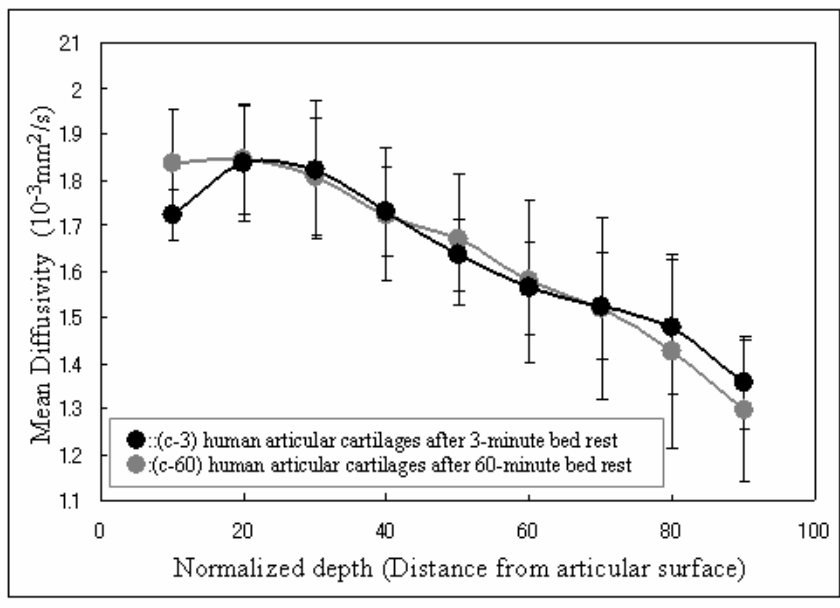

(A) Mean diffusivity

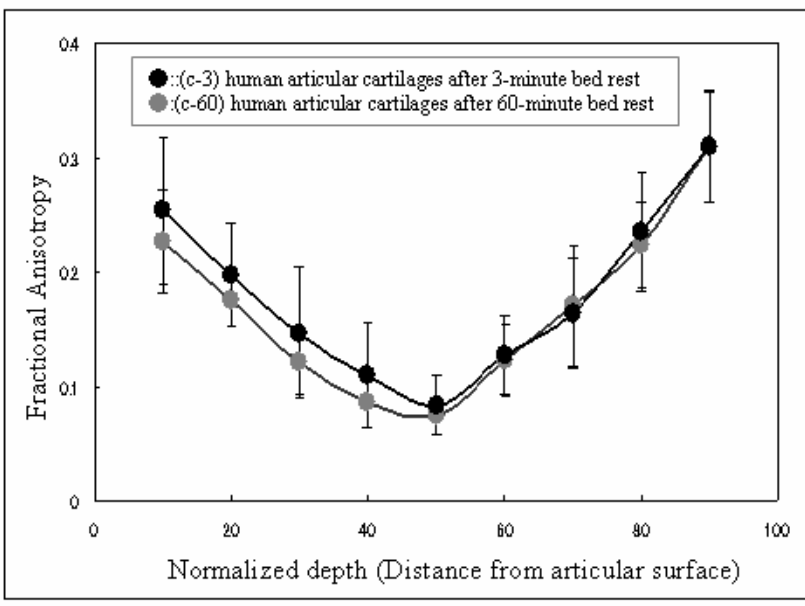

(B) Fractional anisotropy values

Figure.4.

Figure 4. (A) The mean MD values and their standard deviations of (c-3) and (c-60) are shown. The vertical axis indicates $\mathrm{MD}=0.0$ as brightness $=0$ and $\mathrm{MD}$ maximum as brightness $=255$, on the other hand, the horizontal axis indicates the depth from the surface in proportion to the cartilage surface (0) and the subchondral bone interface (100). At the points of (c-3) and (c-60) of human articular cartilage, the MD values at (c-3) were similar to those at (c-60).

(B) The mean FA values and their standard deviations of (c-3) and (c-60) are shown. The vertical axis indicates $\mathrm{FA}=0.0$ as brightness $=0$ and $\mathrm{FA}=1.0$ as brightness $=255$, on the other hand, the horizontal axis indicates the depth from the surface in proportion to the cartilage surface $(0)$ and the subchondral bone interface (100). At the points of (c-3) and (c-60) of human articular cartilage, the FA values were almost similar in the deep layer but slightly higher in the middle and surface 
Table 1. The mean MD values of 5 samples at 9 point between the surface and deep layer of the articular cartilage of (a), (b), (c-3) and (c-60) and their standard deviations were shown.

Table.1.

\begin{tabular}{|c|c|c|c|c|c|c|c|c|c|}
\hline \multirow{3}{*}{ Case } & \multicolumn{9}{|c|}{ Fractional Anisotropy } \\
\hline & \multicolumn{9}{|c|}{ Normalized de pth (Distance from articular surface) } \\
\hline & $10 \%$ & $20 \%$ & $30 \%$ & $40 \%$ & $50 \%$ & $60 \%$ & $70 \%$ & $80 \%$ & $90 \%$ \\
\hline a & $0.070 \pm 0.020$ & $0.089 \pm 0.009$ & $0.133 \pm 0.016$ & $0.173 \pm 0.016$ & $0.223 \pm 0.024$ & $0.277 \pm 0.035$ & $0.326 \pm 0.027$ & $0.391 \pm 0.035$ & $0.460 \pm 0.049$ \\
\hline$b$ & $0.315 \pm 0.043$ & $0.196 \pm 0.015$ & $0.115 \pm 0.021$ & $0.109 \pm 0.039$ & $0.146 \pm 0.046$ & $0.196 \pm 0.073$ & $0.263 \pm 0.094$ & $0.326 \pm 0.072$ & $0.387 \pm 0.068$ \\
\hline$c-3$ & $0.253 \pm 0.063$ & $0.197 \pm 0.045$ & $0.147 \pm 0.057$ & $0.110 \pm 0.045$ & $0.084 \pm 0.026$ & $0.127 \pm 0.035$ & $0.164 \pm 0.048$ & $0.235 \pm 0.062$ & $0.309 \pm 0.048$ \\
\hline$c-60$ & $0.227 \pm 0.045$ & $0.175 \pm 0.023$ & $0.121 \pm 0.027$ & $0.086 \pm 0.023$ & $0.075 \pm 0.016$ & $0.123 \pm 0.030$ & $0.170 \pm 0.054$ & $0.224 \pm 0.038$ & $0.310 \pm 0.049$ \\
\hline
\end{tabular}

Table 2. The mean FA values of 5 samples at 9 point between the surface and deep layer of the articular cartilage of (a), (b), (c-3) and (c-60) and their standard deviations were shown.

Table.2.

\begin{tabular}{|c|c|c|c|c|c|c|c|c|c|}
\hline \multirow{3}{*}{ Case } & \multicolumn{9}{|c|}{ Mean Diffusivity $\left(10^{-3} \mathrm{~mm}^{2} / \mathrm{s}\right)$} \\
\hline & \multicolumn{9}{|c|}{ Normalized depth (Distance from articular surface) } \\
\hline & $10 \%$ & $20 \%$ & $30 \%$ & $40 \%$ & $50 \%$ & $60 \%$ & $70 \%$ & $80 \%$ & $90 \%$ \\
\hline a & $2.338 \pm 0.281$ & $2.120 \pm 0.288$ & $1.964 \pm 0.220$ & $1.822 \pm 0.162$ & $1.637 \pm 0.098$ & $1.467 \pm 0.060$ & $1.320 \pm 0.063$ & $1.191 \pm 0.060$ & $1.063 \pm 0.113$ \\
\hline $\mathrm{b}$ & $1.485 \pm 0.174$ & $1.494 \pm 0.146$ & $1.341 \pm 0.121$ & $1.249 \pm 0.125$ & $1.143 \pm 0.121$ & $1.090 \pm 0.087$ & $1.066 \pm 0.097$ & $0.966 \pm 0.123$ & $0.871 \pm 0.115$ \\
\hline$c-3$ & $1.722 \pm 0.056$ & $1.837 \pm 0.125$ & $1.821 \pm 0.152$ & $1.729 \pm 0.099$ & $1.635 \pm 0.080$ & $1.562 \pm 0.099$ & $1.524 \pm 0.116$ & $1.477 \pm 0.146$ & $1.357 \pm 0.102$ \\
\hline$c-60$ & $1.835 \pm 0.119$ & $1.844 \pm 0.118$ & $1.807 \pm 0.128$ & $1.724 \pm 0.146$ & $1.670 \pm 0.142$ & $1.579 \pm 0.177$ & $1.518 \pm 0.200$ & $1.424 \pm 0.212$ & $1.297 \pm 0.156$ \\
\hline
\end{tabular}

